\title{
Negative Resistance-Based Electronic Impedance Tuner
}

\author{
Y. Zhao, Student Member, IEEE, S. Hemour, Senior Member, IEEE, \\ T. Liu, Senior Member, IEEE, and K. Wu, Fellow, IEEE
}

\begin{abstract}
An electronic impedance tuner using the negative resistance of tunneling diodes is proposed in this paper. Aside from the fact that it is an interesting solution to synthesize impedance with reflection coefficient larger than one, this scheme is proven to be simpler and consume less power than the stateof-the-art techniques. The overall circuit topology consists of two parts, namely, impedance tuning circuit including a hybrid block of PIN and tunneling diode for generating a set of impedance points, and wideband nonlinear transmission line-based $360^{\circ}$ phase shifter for rotating the set of impedance points around the Smith chart from 1.5 to $5 \mathrm{GHz}$. The operating power of the electronic tuner is below $-25 \mathrm{dBm}$, which is limited by the tunneling diode. The worst-case maximum power consumption of the electronic tuner is as low as $3 \mathrm{~mW}$. Such an electronic tuner should be useful for the development of on-wafer noise characterization systems.
\end{abstract}

Index Terms-Electronic tuner, negative resistance, noise measurement, nonlinear transmission line (NLTL), PIN diode, tunneling diode.

\section{INTRODUCTION}

$\mathbf{N}$ EGATIVE resistance can manifest in some electronic devices or circuits, such as tunneling diode, Gunn diode, IMPATT diode, transistor, and operational amplif er with feedback, and so on. The intrinsic amplif cations have long been applied in power amplifie $s$ and oscillators [1], [2]. As a matter of fact, the negative resistance also has potential to be used in the design of electronic impedance tuner to generate specifi impedance with ref ection coeff cient larger than one.

In RF characterization systems, electronic tuner presents a niche area of interest compared with its electromechanical and active counterparts, although it has advantages of small size, low cost, and fast tuning. However, a realizable maximum reflecti $\mathrm{n}$ coeff cient is always limited by its large insertion loss [3]. To reach a larger reflectio coeff cient, one has to compensate the loss by an amplificati $n$. A reflecti $n$ coefficien larger than one means that the reflecte signal is somewhere amplif ed by the circuit. From the standpoint of

Manuscript received August 18, 2017; accepted November 29, 2017. Date of publication December 28, 2017; date of current version February 12, 2018

$\mathrm{Y}$. Zhao and $\mathrm{K}$. Wu are with the Poly-Grames Research Center, Ecole Polytechnique de Montreal, Montreal, QC H3T 1J4, Canada

$\mathrm{S}$. Hemour is with the Laboratoire de $\mathrm{l}^{\prime}$ Integration du Materiau au Systeme, Universite de Bordeaux, 33405 Bordeaux, France $1 \mathrm{VKH}$ RXI\# IHHRU $\square$.

T. Liu is with the Faculty of Electrical Engineering and Computer Science, Ningbo University, Ningbo 315211, China.

Color versions of one or more of the f gures in this paper are available online at http://ieeexplore.ieee.org.

Digital Object Identifie 10.1109/LMWC.2017.2779879

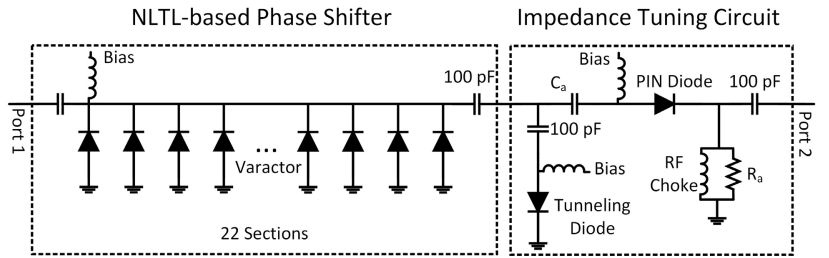

Fig. 1. Diagram of electronic tuner: it consists of a wideband NLTL-based phase shifter and an impedance tuning circuit.
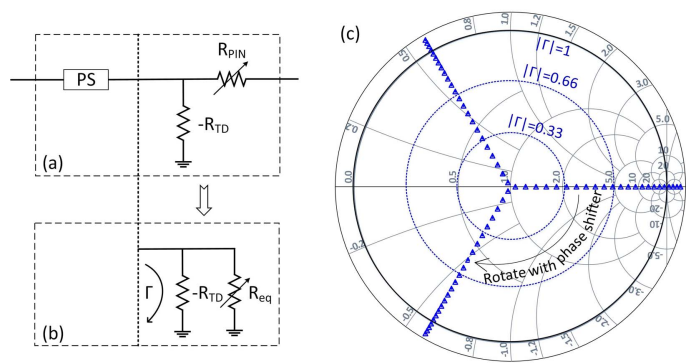

Fig. 2. (a) Idealcircuit of the proposed topology. (b) Simplifie circuit to generate impedance values. (c) Operation principle is illustrated on the Smith chart.

impedance, this implies that the signal has to be ref ected by a negative resistance. The main contribution of this paper is to use the amplif cation property of a negative resistance device to compensate the loss stemmed from electronic tuner. Somehow, it is similar to active tuner, but it is more costeffective due to its simplicity and low power consumption. Interestingly, the very intuitional operation of this circuit could be used for pedagogical purposes.

\section{Methodology}

The proposed topology includes a wideband phase shifter and an impedance tuning circuit (Fig. 1). As depicted in the ideal circuit [Fig. 2 (a)], the core part is a shunt tunneling diode and a series PIN diode. The tunneling diode is biased to the level that presents a negative resistance $\left(-R_{\mathrm{TD}}\right)$. The PIN diode is forward-biased in order to provide a variable positive resistance $\left(R_{\mathrm{PIN}}\right)$.

Without considering any parasitic effect, this topology generates a set of impedance points by a negative resistance in parallel with an equivalent positive resistance $\left(R_{\text {eq }}\right)$ after considering the $50-\Omega$ port effect [Fig. 2(b)]. The total resistance can be calculated by $-R_{\mathrm{TD}} \cdot R_{\mathrm{eq}} /\left(R_{\mathrm{eq}}-R_{\mathrm{TD}}\right)$. Ideally, when $R_{\mathrm{eq}}$ is swept from low resistance (e.g., $50 \Omega$ ) to high resistance (e.g., $600 \Omega$ ), as illustrated in Fig. 2(c), the generated impedance 


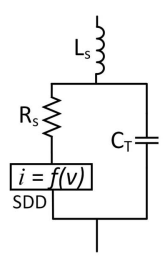

(a)

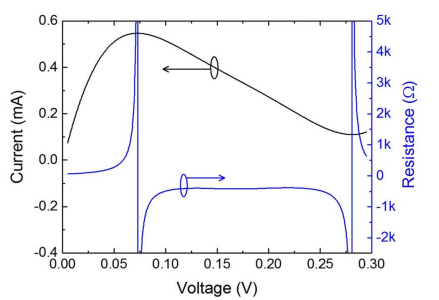

(b)
Fig. 3. (a) Tunneling diode model with SDD. (b) $I-V$ curve and extracted resistance of tunneling diode MBD5057-E28X from MACOM Technology Solutions.

points will be distributed from center to open circuit, then to outside of the Smith chart (negative resistance region).

One criterion to evaluate the electronic tuner is the Smith chart coverage [4], a simple way to improve the coverage is to use a $360^{\circ}$ phase shifter before the impedance tuning circuit. As shown in Fig. 2(c), the set of impedance points generated by the impedance tuning circuit will be rotated by the $360^{\circ}$ phase shifter. With the advantages of broadband, easy impedance matching and low loss, nonlinear transmission line (NLTL) technique is a good solution for controlling the phase shift over a wide frequency range.

However, the tunneling diode and PIN diode are not pure resistance, and parasitic parameters cannot be ignored over $\mathrm{GHz}$ frequency range. The consequence is that the impedance distribution would be distorted. Therefore, two adjusting components, $C_{a}$ and $R_{a}$, can be presented in the impedance tuning circuit in order to adjust the impedance distribution (Fig. 1). The values will be determined according to the operation frequency range as well as the impedance distribution on Smith chart.

\section{Circuit Modeling}

Since parasitic parameters from device and connection pads have impact on the impedance, accurate models of tunneling and PIN diodes should be established prior to the design. A behavioral model, more relevant in f eld operation, is developed from current-voltage $(I-V)$ characteristic and capacitance-voltage $(C-V)$ characteristic as well as measured S-parameters.

It is important to mention that the $I-V$ curve of the tunneling diode is measured with a known positive resistor in parallel with it, in order to avoid unexpected oscillation and irregular discontinuity in the $I-V$ curve [5]. In [Fig. 3(a)], the equation of the $I-V$ curve of the tunneling diode is expressed in a symbolically def ned devices (SDD) model. The seventh-order polynomial is applied to $\mathrm{ft}$ the measured $I-V$ curve [Fig. 3(b)]

$$
\begin{aligned}
i(v)= & -1.5 e-5+0.01879 \cdot v-0.18638 \cdot v^{2} \\
& +0.15171 \cdot v^{3}+7.16518 \cdot v^{4}-45.47 \cdot v^{5} \\
& +110.9539 \cdot v^{6}-97.13563 \cdot v^{7} .
\end{aligned}
$$

The derivation of the $I-V$ equation is also shown in Fig. 3(b). As it can be seen, the resistance remains $-409 \Omega$ in the bias range of 0.1 to $0.25 \mathrm{~V}$. Parasitic parameters

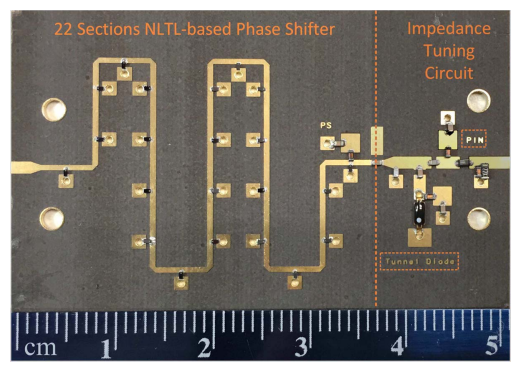

Fig. 4. Photograph of the fabricated electronic impedance tuner.

inductance $L_{S}(0.7 \mathrm{nH})$ related to the long lead, series resistance $R_{S}(0.3 \Omega)$, and total capacitance $C_{T}(0.1 \mathrm{pF})$ are extracted from S-parameter measurements. It is worthwhile to note that there is still no proper model that can represent the RF characteristics of a tunneling diode. The parasitic parameters of tunneling diode extracted in this paper are only approximate.

So far, there is no accurate SPICE model for PIN diode, mainly because it shows distinct features under reverse and forward bias conditions. A PIN diode under forward bias condition can be modeled as a current-controlled series resistance with a parasitic inductance. The series resistance can be extracted from S-parameter measurements under different biases. The PIN diode can be considered as a variable RF resistance from a few $\Omega$ to hundreds of $\Omega$ when current is swept from $\mu \mathrm{A}$ to $\mathrm{mA}$ level. The parasitic inductance is dependent on the package, which is $0.7 \mathrm{nH}$ for SMP1320 with package of SC-79.

\section{EXPERIMENTAL VALIDATION}

The design procedure of an NLTL-based phase shifter has been studied intensively [6], [7]. In this paper, varactor of MA46H120 has been chosen to provide $0.15-1.1-\mathrm{pF}$ capacitance with bias from -15 to $0 \mathrm{~V}$. The distance between each two sections is chosen to be $5.27 \mathrm{~mm}$ so as to have a Bragg cutoff frequency around $6 \mathrm{GHz}$. The fabricated circuit is shown in Fig. 4, where the long transmission line is bended in order to reduce the total circuit size. Twenty-two sections are chosen to realize $360^{\circ}$ phase shift for frequency range $1.5-5 \mathrm{GHz}$.

Ideally, the bias of the tunneling diode could be fxed, and the bias of the PIN diode is swept from 0.3 to $0.8 \mathrm{~V}$, while the bias of phase shifter is swept from -15 to $0 \mathrm{~V}$, in order to generate impedance points all over the Smith chart. However, the parasitic parameters distort the impedance distribution, and a f ne bias of the tunneling diode becomes necessary (swept from 0.14 to $0.7 \mathrm{~V}$ ) to adjust the impedance distribution on Smith chart. The adjusting components $C_{a}$ and $R_{a}$ are chosen as $1.8 \mathrm{pF}$ and $68 \Omega$, respectively, to have a better distribution for frequency range $1.5-5 \mathrm{GHz}$.

For an analog tuner, the impedance can be tuned continually. In other words, there are infi ite impedance states. 25740 impedance states are tested for each frequency with the automatic test program, and the results for different frequencies are shown in Fig. 5. The maximum reflectio coeff cient $\Gamma$ is larger than one for $1.5-1.9 \mathrm{GHz}$ and $4.5-5 \mathrm{GHz}$, while it is in the range of $0.8-1$ for other frequencies. The insertion loss 

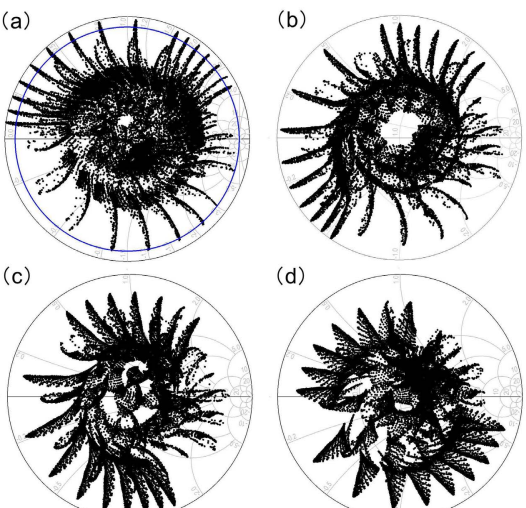

(d)
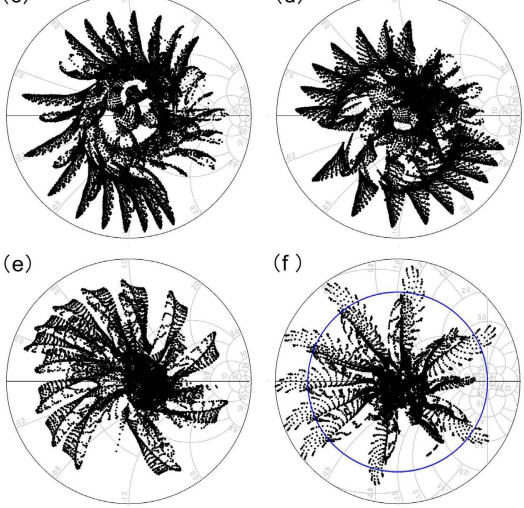

Fig. 5. Measured impedance distribution of electronic tuner at (a) 1.5, (b) 2 , (c) 2.5 , (d) 3, (e) 4, and (f) 5 GHz. The blue-dashed circles in (a) and (f) stand for the ref ection coeff cient which is equal to 1 .

of the phase shifter will reduce the maximum generated by the impedance tuning circuit, which means the maximum $\Gamma$ will decrease after the rotation [Fig. 5(a)]. However, in practice, we only care about the coverage inside the Smith chart without considering the impedance outside of the Smith chart.

The comparison of the proposed electronic tuner with the recent work as well as conventional mechanical tuner are listed in Table I. The proposed tuner presents the largest maximum $\Gamma$ than other work, which can be used in the noise characterization system for active device with impedance near $0 \Omega$. It also presents the lowest power consumption, which is mainly consumed by the tunneling diode and PIN diode. Limited by the tunneling diode, the maximum operation power of the proposed tuner is around $-25 \mathrm{dBm}$, which is lower than the MOS FET and PIN diode-based electronic tuner and the mechanical tuner. However, this power level still can be used for noise measurement, since the output power of the noise source is always below $-50 \mathrm{dBm}$. Further work can be done to improve the power handling capability. For instance, two transistors and linear positive resistors could be combined to generate large power negative resistance [10].

It should be noted that the proposed electronic tuner requires precise bias sources for both PIN diode and tunneling diode. Small variation of biases may lead to the shift of impedance. Repeatability is presented to describe the stability of tuner, which denotes the difference between two S-parameter measurements. The repeatability test in this paper is implemented by measuring 100 impedance states for 10 times with an interval of $10 \mathrm{~min}$. As listed in Table I, the repeatability of the proposed tuner is around $25 \mathrm{~dB}$, which is limited by the stability and precision of the bias sources.

Over the GHz frequency range, the distorted impedance distribution, caused by the parasitic parameters, can be corrected for a different frequency range by replacing the adjusting components. Small footprint devices and shorter connection
TABLE I

COMPARISON With THE STATE-OF-THE-ART WORK

\begin{tabular}{c|c|c|c|c}
\hline & {$[8]$} & {$[4]$} & {$[9]$} & This work \\
\hline $\begin{array}{c}\text { Frequency } \\
\text { band (GHz) }\end{array}$ & $130-170$ & $0.3-0.8$ & $0.8-8$ & $1.5-5$ \\
\hline $\begin{array}{c}\text { Smith chart } \\
\text { coverage }\end{array}$ & Small & High & High & High \\
\hline $\begin{array}{c}\text { Nonlinear } \\
\text { device }\end{array}$ & MOS FET & PIN diode & $\begin{array}{c}\text { Mechanical } \\
\text { probe }\end{array}$ & $\begin{array}{c}\text { Varactor, } \\
\text { tunnel diode } \\
\text { PIN diode }\end{array}$ \\
\hline Maximum $\Gamma$ & 0.5 & 0.87 & $0.9-0.98$ & $>1$ \\
\hline $\begin{array}{c}\text { Power } \\
\text { handling }\end{array}$ & $8 \mathrm{dBm}$ & $>30 \mathrm{dBm}$ & $1414 \mathrm{~W}$ & $-25 \mathrm{dBm}$ \\
\hline $\begin{array}{c}\text { Power con- } \\
\text { sumption }\end{array}$ & - & Medium & High & Low $(3 \mathrm{~mW})$ \\
\hline Loss & $6-22 \mathrm{~dB}$ & $<2 \mathrm{~dB}$ & $<1 \mathrm{~dB}$ & $2-25 \mathrm{~dB}$ \\
\hline Size & Small & Medium & Large & Medium \\
\hline Repeatability & - & - & $45 \mathrm{~dB}$ & $25 \mathrm{~dB}$ \\
\hline
\end{tabular}

pads can be chosen to further reduce the parasitic effects so as to improve the performance. In practice, the high insertion loss of the proposed electronic tuner will be calibrated prior to the noise measurement.

\section{CONCLUSION}

In this paper, we illustrate the potential use of a negative resistance device in the development of an electronic impedance tuner. The experiments exhibit a good performance of the electronic tuner over the frequency range $1.5-5 \mathrm{GHz}$. The proposed topology can be an alternative solution for on-wafer noise characterization systems. Although only parts of frequencies can have impedance states with refection coeff cient larger than one, accurate modeling of tunneling diode and PIN diode can be further investigated to predict the impedance distribution eff ciently. In addition, large power negative resistance devices can be studied to have electronic tuners work for high-power condition.

\section{REFERENCES}

[1] M. E. Hines, "Negative-resistance diode power amplification" IEEE Trans. Electron Devices, vol. ED-17, no. 1, pp. 1-8, Jan. 1970.

[2] M. E. Hines, "High-frequency negative-resistance circuit principles for Esaki diode applications," Bell Syst. Tech. J., vol. 39, no. 3, pp. 477-513, May 1960.

[3] F. M. Ghannouchi and M. S. Hashmi, Load-Pull Techniques With Applications to Power Amplifier Design, vol. 32. Dordrecht, The Netherlands: Springer, 2012.

[4] C. Sanchez-Perez, J. de Mingo, P. L. Carro, and P. Garcia-Ducar, "Design and applications of a 300-800 MHz tunable matching network," IEEE J. Emerg. Sel. Topics Circuits Syst., vol. 3, no. 4, pp. 531-540, Dec. 2013.

[5] HP 4142B Modular DC source/Monitor Practical Applications-High Speed DC Characterization of Semiconductor Devices from Sub pA to 1A, Hewlett-Packard, Palo Alto, CA, USA, 1987.

[6] A. S. Nagra and R. A. York, "Distributed analog phase shifters with low insertion loss," IEEE Trans. Microw. Theory Techn., vol. 47, no. 9, pp. 1705-1711, Sep. 1999

[7] K. Payandehjoo, "Study of nonlinear transmission lines and their applications," Ph.D. dissertation, Dept. Elect. Comput. Eng., McGill Univ., Montreal, QC, Canada, 2006.

[8] S. Bouvot et al., "A D-band tuner for in-situ noise and power characterization in BiCMOS $55 \mathrm{~nm}$," in Proc. IEEE 17th Topical Meeting Silicon Monolithic Integr. Circuits RF Syst. (SiRF), Jan. 2017, pp. 103-106.

[9] Focus Microwave Inc. CCMT-808. Accessed: 2017. [Online]. Available: https://focus-microwaves.com/data-sheet-library/

[10] L. Chua, J. Yu, and Y. Yu, "Bipolar-JFET-MOSFET negative resistance devices," IEEE Trans. Circuits Syst., vol. CAS-32, no. 1, pp. 46-61, Jan. 1985 